normalized and equally weighted. We compared the multiple index of deprivation between immigrants and the local population. We also measured access to primary healthcare services, as the nearest distance from the centroid of census tract to the corresponding health service through a GIS platform.

Results Socioeconomically deprived immigrants concentrate in the same areas that as the local population, that is in the north and centre boroughs of the city. These deprived areas do not only concentrate higher deprived population, but also concentrate higher crime rates and fewer healthcare services than other areas of the city. Nevertheless, the spatial access to primary healthcare services is similar across the four primary healthcare services, averaging 1 kilometer of distance.

Conclusion The spatial characterization of socioeconomically deprived population and their distance to healthcare services is useful for policy design and evaluation. We found that both immigrant and local population in socioeconomic deprivation tend to concentrate in similar poorer sectors of the city. The multidimensional index of deprivation was measured for the first time in Chile and became highly informative. More studies are required to understand how socioeconomic deprivation might impact on accessing healthcare among immigrant population in Chile and other countries.

\section{P50 ABSTRACT WITHDRAWN}

\section{P51 COMORBIDITIES AND THE CLINICAL PATHWAY TO ACCESS JOINT REPLACEMENT SURGERY: AN EXPLORATORY QUALITATIVE STUDY}

\begin{abstract}
${ }^{1,2} \mathrm{~B}$ Podmore* ${ }^{1,2} \mathrm{~A}$ Hutchings, ${ }^{1} \mathrm{MA}$ Durand, ${ }^{3} \mathrm{~J}$ Robson, ${ }^{4} \mathrm{~S}$ Konan, ${ }^{1,2} \mathrm{~J}$ van der Meulen, ${ }^{1} \mathrm{R}$ Lynch. ${ }^{1}$ Health Services Research and Policy, London School of Hygiene and Tropical Medicine, London, UK; ${ }^{2}$ Clinical Effectiveness Unit, The Royal College of Surgeons of England, London, UK; ${ }^{3}$ Centre for Primary Care and Public Health, Queen Mary University of London, London, UK; ${ }^{4}$ University College London Hospitals NHS Foundation Trust, London, UK
\end{abstract}

\subsection{6/jech-2018-SSMabstracts. 175}

Background Hip and knee replacement surgery is one of the most common and effective surgical procedures. The rise in multi-morbidity world-wide is leading to increasing numbers of patients with comorbid conditions undergoing joint replacement surgery. Financially stretched commissioners of health care services in the English National Health Service (NHS) are increasingly seeking to restrict access to elective surgery, including hip and knee replacement surgery despite a lack of evidence to support these decisions. It is important to get a better understanding of the referral and selection of patients with comorbidities for joint replacement surgery.

Methods An exploratory qualitative approach involving semistructured interviews with eight orthopaedic surgeons, seven general practitioners (GPs), and five professionals working in intermediate musculoskeletal services (specific centres within the English NHS to support the referral process from primary to specialised care).

Results In general, the presence of comorbidities was not seen as a barrier to being referred or selected for joint replacement. Each professional group, however, concentrated on different aspects of the patients' condition which appeared to affect how each group managed patients with comorbidities. GPs focused on the long-term impact that comorbidities have on the patients' everyday life. Intermediate care professionals focused on the short-term impact of comorbidities on the patients' likelihood of being selected for surgery. Orthopaedic surgeons focused on the short-term impact of comorbidities on the surgery itself. This implied there was a disagreement about roles and responsibilities in the management of patients with comorbidities. None of the three groups believed it was their responsibility to address comorbidities in preparation for surgery. This disagreement was identified as a reason why some patients seem to 'get lost' in the referral system when they were considered to be unprepared for surgery. Patients were then potentially left to manage their own comorbidities before being reconsidered for joint replacement.

Conclusion At the clinician-level, comorbidities were not perceived as a barrier to accessing joint replacement surgery but at the pathway-level, it may create an implicit barrier such that patients with comorbidities may get 'lost' to the system. The current orthopaedic clinical pathway may be less suitable for patients with comorbidities.

\section{P52 THE EFFECT OF STEP-DOWN INTERMEDIATE CARE ON DAYS LOST TO DELAYED DISCHARGE FROM HOSPITAL: A CONTROLLED INTERRUPTED TIME SERIES ANALYSIS}

KA Levin*, E Crighton. Public Health Directorate, NHS GGC, Glasgow, UK

\subsection{6/jech-2018-SSMabstracts. 176}

Background Step-Down Intermediate Care (IC) was developed in Glasgow City and implemented in November 2014. IC units act as a bridging service between hospital and home, for those deemed medically fit for discharge, but who would otherwise spend some time delayed in hospital, usually due to a lack of appropriate care, support or accommodation in the community. The aim of this study was to measure the effect of IC on days delayed.

Methods Rate of days delayed per 1000 population aged 75 years + in Glasgow City was compared before and after onset of IC with a 6 month phase-in period, using segmented linear regression with 23-month (January 2013-November 2014) pre- and 13-month (June 2015-June 2016) post- intervention periods. Rate of delayed days for residents of Inverclyde and West Dunbartonshire (I\&WD)- areas with similar high levels of deprivation, within NHS GGC, but with no IC in place were used as a comparison group, as in April 2015 a national target was set to reduce delays.

Results Between January 2013 and November 2014 rate of days delayed in hospital increased by $41 \%$. Rate of days delayed in I\&WD was approximately half that of Glasgow at the start of the study period, however these also increased in the pre intervention period, by 33\%. Rates reduced in both areas between November 2014 and June 2015. After accounting for secular changes pre-intervention, Glasgow City saw a level change of $-57.66(-60.85,-54.48)$ days per 1000 population with no significant change in trend, $0.35(-0.19,0.88)$. However, following adjustment for changes in I\&WD, and therefore a factoring out of the new national target, the impact of IC in Glasgow City was found to be a level change of $-12.56(-17.65,-7.47)$ and a trend change of -0.38 $(-0.73,-0.04)$. This is equivalent to a predicted reduction due to IC of -7.28 days delayed per 1000 popn, in June 2016 , and a relative reduction of $18 \%$. Rate of days delayed in Glasgow City continued to increase over time after the 
introduction of IC, though at a slower rate than if IC had not existed.

Conclusion The introduction of a national target resulted in a reduction of delayed days. After factoring out this reduction, IC was responsible for a further reduction in delayed days of $18 \%$. The rate of days delayed continued to increase over time after the introduction of IC, though at a slower rate.

\section{P53 THE ASSOCIATIONS BETWEEN COMMON MENTAL DISORDERS (ANXIETY AND DEPRESSION) AND SOCIO- ECONOMIC AND DEMOGRAPHIC FACTORS IN TWO RUSSIAN CITIES}

${ }^{1} \mathrm{~S}$ Cook*, ${ }^{2} \mathrm{AV}$ Kudryavtsev, ${ }^{1} \mathrm{~N}$ Bobrova, ${ }^{3,4} \mathrm{~S}$ Malyutina, ${ }^{1,5} \mathrm{DA}$ Leon. ${ }^{1}$ Department of NonCommunicable Disease Epidemiology, London School of Hygiene and Tropical Medicine, London, UK; ${ }^{2}$ Central Scientific Research Laboratory, Northern State Medical University, Arkhangelsk, Russia; ${ }^{3}$ Research Institute of Internal and Preventive Medicine - Branch of ICandG SB RAS, Novosibirsk, Russia; ${ }^{4}$ Novosibirsk State Medical University, Ministry of Health of Russia, Novosibirsk, Russia; ${ }^{5}$ Department of Community Medicine, Arctic University of Norway, UiT, Tromsø, Norway

\subsection{6/jech-2018-SSMabstracts.177}

Background Common mental disorders (anxiety and depression) are important public health concerns worldwide. There is very little evidence about the risk factors for these in Russia despite high rates of associated markers: suicide and alcohol-attributable mortality. The aim of this study was to investigate the associations between common mental disorders and socio-demographic factors in the Russian adult population.

Methods Data were from two population-based cross-sectional surveys conducted in 2016-17 among men and women aged 35-69 years old resident in the Russian cities of Arkhangelsk and Novosibirsk as part of the International Project on Cardiovascular Disease in Russia (IPCDR). Participants were recruited and interviewed in their homes. Information collected included questions on socio-demographic factors (marital status, education, employment and financial status). Depression was defined as a score of $\geq 10$ on the PHQ-9 instrument and anxiety as a score of $\geq 10$ on the GAD-7 instrument. Financial status was on a 5-point scale from not enough money for food to no financial constraints. Logistic regression was used to estimate effects of socio-demographic factors on anxiety and depression adjusting for age, sex and city and then for all other socio-demographic variables. Volume of alcohol consumed per year and problem drinking defined as CAGE score $\geq 2$ were entered into the models at the last step in order to assess the effect on the associations of interest.

Results After adjustment for all socio-demographic variables depression was more prevalent among women (OR 1.93 95\% CI 1.53, 2.44), those who were divorced or separated compared to married (OR $1.4695 \% \mathrm{CI} 1.10,1.92$ ), and not in regular employment (OR 1.49 95\% CI 1.18, 1.88). There was a monotonic increase in risk of depression across the 5 categories of financial status from poorest to most affluent ( $\mathrm{p}$-value trend $\leq 0.001$ ), with an OR $>6$ from bottom vs top category. Anxiety was more prevalent in women (OR 1.93 95\% CI 1.42, 2.62). As with depression financial status also showed a strong inverse trend in risk $(\mathrm{p}<0.001)$. Education was inversely associated with depression and anxiety after adjustment for age, sex, and city but not after adjustment for other socio-demographic variables. The pattern of results remained similar after additional adjustment for alcohol use.

Conclusion Degree of financial hardship was strongly associated with symptoms of anxiety and depression even after adjustment for education, employment status and alcohol use. These findings underline the importance of considering social circumstances in addressing poor mental health.

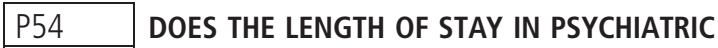 INPATIENT UNITS AFFECTS THE SOCIAL INTEGRATION OF SEVERE MENTALLY-ILL PATIENTS? A STUDY IN FIVE EUROPEAN COUNTRIES}

P Smith*, P Nicaise, V Lorant. Institute of Health and Society, Université Catholique de Louvain, Brussels, Belgium

10.1136/jech-2018-SSMabstracts. 178

Background During the last three decades, in high-income countries, a transition from institutional psychiatric care to community care and a reduction in the hospital length of stay has taken place to provide community care and promote patients' social integration. However, the impact of length of stay in psychiatric inpatient unit on the different dimensions of patients' social integration remains unclear. The aim of this study is to determine whether longer length of stay in psychiatric inpatient unit decreases the social integration of Severe Mentally-Ill (SMI) patients.

Methods Within the European COFI study (prospective cohort study), data were collected for 7302 SMI patients hospitalised in 2015 in the UK, Italy, Germany, Poland and Belgium. Social integration was measured using the SIX index in baseline and at one year of follow-up. The SIX index includes the following dimensions of social integration: employment, housing, living situation and contacts with friends. Correlation and regressions models were performed to test the association between length of stay in psychiatric inpatient unit and patients' social integration.

Results The average score of social integration of SMI patients in baseline was $3.8 / 6 \quad(\mathrm{SD}=1.39)$ and decreased by -0.14 $(\mathrm{SD}=1.29)$ one year later. We found a small but significant negative correlation between length of stay in hospital and social integration of patients at one year $(\mathrm{r}=-0.04, \mathrm{p}=0.03)$. This association remained significant when adjusting for patient characteristics and hospitals as a random intercept. At one year, the most correlated dimensions of social integration with length of stay were housing $(\beta=-6.3, p<0.0001)$ and employment $(\beta=-1.8, \mathrm{p}=0.01)$.

Conclusion This study supports the importance of policies and interventions to reduce the length of stay in psychiatric inpatient unit for SMI patients to preserve their social integration. Housing and employment are the main dimensions of social integration negatively impacted by length of stay. Therefore, special attention must be given to help SMI patients to find and retain housing and employment during psychiatric hospitalisations. 